\title{
Solitary staphylococcal liver abcess in a neonate Das $S^{1}$, Paul DK', Chatterjee $A^{3}$, Raut $S^{4}$
}

\begin{abstract}
:
A 28 days old neonate presented with high fever, abdominal distension, poor feeding and lethargy. Sepsis screen was positive; ultrasound and computed tomography of the abdomen demonstrated a multiloculated hepatic abcess in the right lobe of the liver. The baby was treated with intravenous antibiotics for 6 weeks and percutaneous aspiration of the abcess, resulting in excellent recovery.
\end{abstract}

Keywords: liver abcess; Staphylococcus aureus; percutaneous aspiration; empiric antibiotics

Introduction- Neonatal liver abcess is a rare entity associated with high morbidity and mortality. The diagnosis needs a high index of suspicion because liver abcess presents with non specific signs and symptoms, which are essentially those of neonatal sepsis. Majority results from ascending haematogenous infections via portal and umbilical veins, via biliary tree or direct contiguous spread from neibouring structures ${ }^{1}$. Major risk factors are blood culture proven sepsis, incorrectly placed umbilical catheter and omphalitis. Minor risk factors are necrotizing enterocolitis, exchange transfusion, perinatal asphyxia, gastrochisis requiring surgery, infant of diabetic mother, ventriculo-peritoneal shunt, and neutrophil function defects. Liver abcess in neonates can be either single or multiple. Single abcess are often large, well localized, and drainable by surgical methods, have a subacute course and preceded by umbilical infections. Multiple abcesses are small in size, not drainable by surgical techniques, follow a fulminant course and are not due to umbilical infections ${ }^{2}$. Most common causative organisms are Staphylococcus aureus, Streptococcus pyogenes, Eschericia coli, whereas Klebsiella, Pseudomomas, Corynebacterium acnes, anaerobes, Candida species are less commonly encountered. $50 \%$ of cases have polymicrobial etiology ${ }^{3}$.
Case report- A 28 days old male term neonate was admitted with history of history of high grade fever, progressive abdominal distension, lethargy, poor feeding and vomiting for past 2 days. His mother had an uneventful antenatal and intranatal periods. Normal vaginal delivery ensued with Apgar scores being $9 / 10$ and $10 / 10$ at 1 and 5 minutes after birth. He was exclusively breastfed and no prelacteal feeding was given.

On examination, the baby was febrile (temperature $102 \mathrm{~F}$ ), tachycardic (heart rate of 180/minute), tachypnoeic (respiratory rate-60/minute), and capillary refill time of 2 seconds. The neonatal reflexes were poorly elicitable. The liver was firm and palpable 6 centimeters, below the right costal margin along the right midclavicular line with a span of 9 $\mathrm{cm}$. The left lobe was palpable. Spleen was palpable $2 \mathrm{~cm}$ below the costal margin along the splenic axis. A lump in the hypochondrium $3 \times 4 \mathrm{~cm}$, firm, smooth surface, immobile was felt separately from the liver. The sepsis screen was positive with $22 \%$ bandemia, $16 \mathrm{~mm}$ micro ESR in one hour. Ultrasound of the abdomen showed hepatosplenomegaly and presence of a hypoechoeic lesion with inhomogenous areas in the right lobe of the liver. There was no ascites and splenoportal venous system was normal in colour doppler study. Computed tomography (CT) of the

1. Dr. Suman Das, Residential Medical Officer, Department of Pediatrics, Dr. B C Roy Post Graduate Institute of Pediatric Sciences.

2. Dr. Dilip Kumar Paul, Professor, Department of Pediatrics, Dr. B C Roy Post Graduate Institute of Pediatric Sciences.

3. Dr. Anish Chatterjee, AssociateProfessor, Department of Pediatrics, Dr. B C Roy Post Graduate Institute of Pediatric Sciences.

4. Dr. Sumantra Raut, Residential Medical Officer, Department of Pediatrics, Dr. B C Roy Post Graduate Institute of Pediatric Sciences.

Corresponds to: Dr. Suman Das, 44, Talpukur Road, Deulpara, Naihati, North 24 Parganas, West Bengal, India, PIN- 743165. Email- dr.sumands@gmail.com 


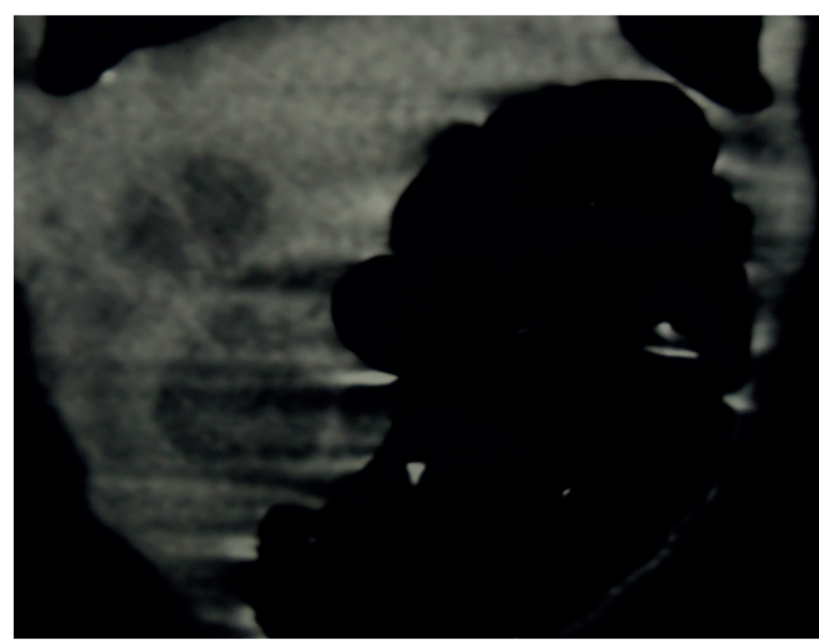

Fig 1- The coronal section of computed tomography of abdomen image shows the bilobed abcess (marked by block arrows), and internal septation in both the lobes of the abcess (marked by line arrow).

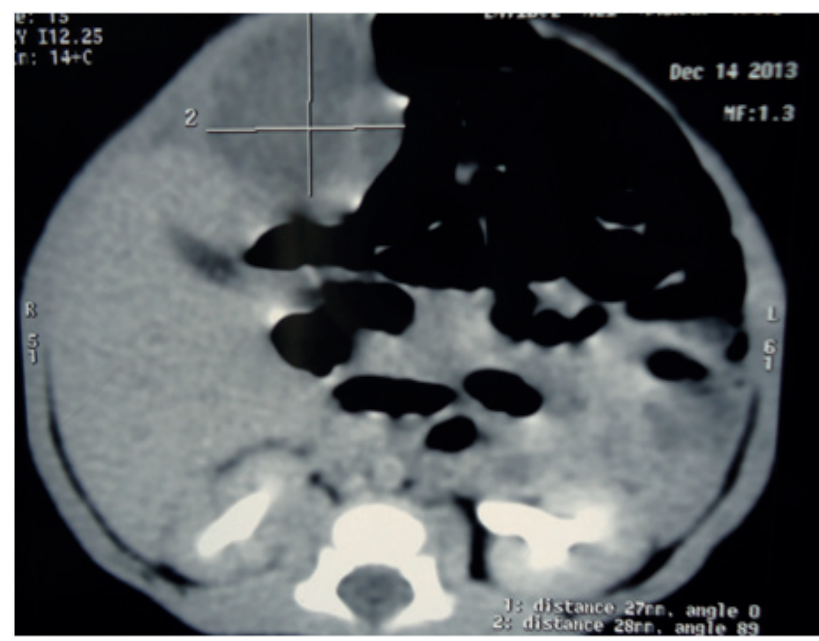

Fig.2- The computed tomography of abdomen in axial cut shows the abcess cavity in the right lobe of the liver.

abdomen revealed bilobed multiloculated lesion $(47 \times 30 \times 28 \mathrm{~mm})$ with enhancing margins involving segments IV and VIII (Fig 1,2). Common bile duct was normal in course and caliber; intrahepatic biliary radicles were not dilated. The lung bases and were pleural spaces were clear. Prothrombin time (PT) was 13.4 seconds (control 11.2 seconds), INR- 1.2, Activated partial prothrombin time (APTT) - 28 seconds (control-26 seconds). Blood culture was sterile and amoebic serology (Indirect haemagglutination assay) was negative.

Confirming the diagnosis as hepatic abcess, we started intravenous antibiotics Meropenem and Vancomycin empirically. Ultrasound guided percutaneous aspiration of $2.5 \mathrm{ml}$ pus from the abcess cavity was done. The pus culture revealed Staphylococcus aureus sensitive to the empiric antibiotics. Repeated ultrasound abdomen at weekly intervals showed gradual reduction in the abcess size. Fever spikes gradually reduced and the baby became afebrile by $20^{\text {th }}$ day of antibiotic therapy. On day 42 of treatment ultrasound revealed few scattered calcific foci in the hepatic parenchyma, without definite evidence of residual abcess. The baby was discharged 50 days after hospital admission when he was found to have steady weight gain of 25-30 grams/ day for 7 consecutive days.

Discussion- Neonatal liver abcess is a very rare entity ${ }^{4,5}$ The first case reported was in 1936, when 2 neonates with fatal peritonitis and solitary liver abcess at necropsy were reported ${ }^{6}$. In 2005, Tan et al reported 6 cases of neonatal liver abcess and stated that there are fewer than 100 cases in English literature from 1936 to $2005^{3}$. Since that report, additional 21 cases have been reported till $2013{ }^{6}$. In January 2014, Fysal et al reported another case of neonatal liver abcess ${ }^{4}$. Thus only 28 cases have been reported from 2005 to January 2014.Increasing trend of this rare condition have been reported among babies admitted in neonatal intensive care units and among preterm babies ${ }^{4}$.

The treatment comprises of correcting the predisposing conditions (withdrawal of umbilical catheter), percutaneous drainage for solitary abcess or open drainage for multiple abcesses and or large abcess ${ }^{7}$. Percuteneous drainage techniques have been found to be safe and efficacious in children, but experience in neonates is limited ${ }^{8}$. Open surgical exploration has an advantage of possible treatment of an intra-abdominal source of infection ${ }^{8}$. However based on their 14 years experience Lee et al stated that neonatal liver abcess is associated with good long term outcome and minimal complications when imaging guided percutaneous guided aspiration or drainage is performed in conjunction with long term antibiotic coverage ${ }^{9}$. Parenteral antibiotics based on culture sensitivity pattern should be given for 6-8 weeks. In culture negative cases, broad-spectrum antibiotics covering both gram positive and negative organisms should be instituted ${ }^{7}$.

As an imaging modality, ultrasound has the following limitations- it is less sensitive in detecting small multiple abcess. Moreover findings on ultrasound are not diagnostic for hepatic abcess. Differential diagnosis includes hepatoblastoma, infantile haemangioendothelioma, mesenchymal hamartoma when the lesion is solitary and secondaries of neuroblastoma if lesions are multiple ${ }^{10}$. CT scan is 
diagnostic, the characteristic appearance being a well circumscribed, low attenuating mass with contrast enhancing rim ${ }^{10}$. Multiloculation is an important sign when present, as in our case.

Articles have reported fatal peritonitis ${ }^{6}$, pyopericardium ${ }^{11}$, portal vein thrombosis and portal cavernoma formation ${ }^{7,10}$ as complications associated with hepatic abcess in neonates.

All previous reported cases were associated with one or more risk factors. The occurrence of liver abcess in a neonate without any identifiable risk factor, the success of percutaneous aspiration in treating the case and recovery without any complication are the remarkable features of this case.

\section{Reference}

1) Simeunovic E, Arnold M, Sidler D, Moore SW. Liver abcess in neonates. Pediatr Surg Int 2009 Feb;25(2):153-156. http://dx.doi.org/10.1007/s00383-008-2307-5

2) Sharma S, Mohta A, Sharma P. Hepatic abcess in a preterm neonate. Indian Pediatr 2007;44:226-228.

3) Tan NWH, Sriram B, Tan-Kendrick APA, Rajadurai VS. Neonatal hepatic abcess in preterm infants: a rare entity. Ann Acad Med Singapore 2005;34:558-564.

4) Fysal N, Mohammed Saleem EK, Kutty PM. Liver abcess in a neonate- a rare entity. Annals Medicine 2014 ;1 (1):2629.

5) Lee SH, Tomlinson C, Temple M, Amaral J, Connoly BL. Liver abcess in neonates. Pediatr Surg Int 2009;25:153-156. http://dx.doi.org/10.1007/s00383-008-2307-5

6) Bosnali O,MoraligluS,CelayerCerrah A, Paktas O. Liver abcess: increasing occurrence in preterm newborn. $J$ Neonatal Surg 2013;(2):23.
7) Shah I, Bhatnagar S. Liver abcess in a newborn leading to portalvein thrombosis. IndianJPediatr 2009;76:1268-1269. http://dx.doi.org/10.1007/s12098-009-0244-5

8) Vachon L, Diament MJ, Stanley P. Percutaneous drainage of hepatic abcess in children. J PediatrSurg 1986;21:366-368. http://dx.doi.org/10.1016/S0022-3468(86)80206-8

9) Lee SH, Tomlinson C, Temple M, Amaral J, Connolyb BL. Imaging guided percutaneous needle aspiration or catheter drainage of neonatal liver abces- a 14 years experience. Am J Roentgenol 2008;190:616-622. http://dx.doi.org/10.2214/AJR.07.2888

10) Aggarwal S, Mathur NB, Garg A. Portal vein thrombosis complicating neonatal hepatic abcess. Indian Pediatr 2003;40:997-1001.

11) Ravisankaran P, Rajamani G. Liver abcess with pyopericardium. Laparoscopic management in a preterm neonate. J Indian Assoc Pediatr Surg 2010;15:72-73. http://dx.doi.org/10.4103/0971-9261.70648 